\title{
Troca Estratosfera-Troposfera como uma fonte de Ozônio para a Camada Limite Planetária
}

\author{
Stratosphere-Troposphere Exchange as an Ozone source to Planetary Boundary \\ Layer
}

immediate

\author{
Letícia de Oliveira dos Santos, Lucas Vaz Peres, Franciano Scremin Puhales, Vagner Anabor e \\ Damaris Kirsch Pinheiro \\ Universidade Federal de Santa Maria \\ deoliveiraaicitel@gmail.com; lucasvazperes@gmail.com; franciano.puhales@ufsm.br; vanabor@gmail.com; damaris@ufsm.br
}

\begin{abstract}
Resumo
Foram identificados eventos de Troca Estratosfera-Troposfera (TET) sobre a região Sul do Brasil que atuaram como fonte de ozônio estratosférico para a Camada Limite Planetária (CLP) durante 3 anos (2011-2013). Ocorreram 13 eventos com influência direta entre $29^{\circ}$ e $31^{\circ} \mathrm{S}$ (Região central do Rio Grande do Sul) com aumento na coluna total de ozônio. Destes, 4 casos ocorreram em 2011, 5 em 2012 e 4 em 2013. Eles foram divididos: em relação à latitude da troca, atuação ou não da Corrente de Jato em altos níveis e altitude de origem e chegada das parcelas de ar. As parcelas de ar cruzam a tropopausa entre 120 e $320 \mathrm{hPa}$ (tropopausa dinâmica), entrando até a baixa troposfera. Grande parte dos casos (30,8\%) chegou à 1000 hPa e o restante entre 600 e 900 hPa. Apenas em um dia a TET aconteceu em latitude menor que $29^{\circ} \mathrm{S}$; nos outros (92,3\%) a TET ocorreu em latitudes maiores que $31^{\circ} \mathrm{S}$ (quanto mais perto do pólo, maior a concentração de ozônio, salvo exceções de influência secundária do Buraco de Ozônio) ou entre $29^{\circ}$ e $31^{\circ} \mathrm{S}$. Na maior parte dos casos (61,5\%), observou-se a TET em conjunto com a ação do núcleo da corrente de jato. Palavras-chave: Troca Estratosfera-Troposfera, Ozônio, Camada Limite Planetária.
\end{abstract}

\begin{abstract}
Stratosphere-troposphere exchange (STE) events were identified over southern Brazil acting as a stratospheric ozone source to the Planetary Boundary Layer (PBL) during 2011-2013 period. There were 13 events with direct influence between 29 and $31^{\circ} \mathrm{S}$ (center of Rio Grande do Sul), with increase in ozone total column. In these cases, 4 occurred in 2011, 5 in 2012 and 4 in 2013. They were divided: in relation to the exchange latitude, the upper-level Jet Stream act, altitude of source and arrive of air parcels. The air parcels cross the tropopause between 120 and $320 \mathrm{hPa}$ (dynamic tropopause), entering troposphere until the lower troposphere. Most cases (30,8\%) reached $1000 \mathrm{hPa}$ and the rest between 600 and $900 \mathrm{hPa}$. Just in one day the STE occurred in a lower latitude than $29^{\circ} \mathrm{S}$; in all the other days (92,3\%), STEs occurred in higher latitudes than $31^{\circ} \mathrm{S}$ (the closer it gets to the pole, the bigger is the ozone concentration, except in Ozone Hole Influence events) or between 29 and $31^{\circ} S$. In most cases $(61,5 \%)$ it was observed STE along with the Jet Streak act.
\end{abstract}

Keywords: Stratosphere-Troposphere Exchange, Ozone, Planetary Boundary Layer. 
analisadas (Appenzeler et al., 1996).

Como a concentração do $\mathrm{O}_{3}$ varia com a latitude, foi também considerada a latitude onde aconteceu a entrada do ar da estratosfera para a troposfera, que posteriormente influencia a coluna total na região analisada. Foram consideradas latitudes mais altas aquelas maiores que $31^{\circ} \mathrm{S}$, latitudes mais baixas aquelas menores que $29^{\circ} \mathrm{S}$ e latitudes iguais à região analisada entre 29 e $31^{\circ} \mathrm{S}$.

Com os dados de concentração de $O_{3}$, foi feita a média em relação aos 5 (cinco) dias anteriores ao evento (TET). Este valor foi subtraído do valor da concentração diária de $\mathrm{O}_{3}$ no dia do evento e, finalmente, o valor obtido desta subtração foi dividido pela média dos 5 dias anteriores. $\mathrm{O}$ resultado foi a variação (aumento ou diminuição) da coluna total de $O_{3}$ para a latitude de $29,4^{\circ} \mathrm{S}$ em relação aos 5 dias anteriores.

Neste trabalho foram consideradas apenas as TETs profundas, ou seja, aquelas que tiveram uma extensão vertical (variação do nível vertical em coordenadas isobáricas) maior ou igual a $350 \mathrm{hPa}$ desde a passagem pela tropopausa até seu destino final (Skerlak et al., 2015). Além disso, somente os casos em que a variação na coluna total de ozônio aumentou foram contabilizados, obtendo assim a influência das TETs como fonte de ozônio para a baixa troposfera.

\section{Resultados e Discussões}

Foram analisadas as TETs profundas que aconteceram entre os anos de 2011 e 2013 e influenciaram diretamente a entrada de ar estratosférico rico em ozônio para a CLP da região central do Rio Grande do Sul, totalizando 3 anos de análise.

Ocorreram 13 eventos de TET profunda entre $20 \mathrm{e}$ $50^{\circ} \mathrm{S}$ que vieram influenciar a região de interesse (entre 29 e $31^{\circ} \mathrm{S}$ ), aumentando o conteúdo de ozônio na camada.

A tabela 1 traz os detalhes destes eventos, tais como as latitudes que ocorreram as trocas, a atuação ou não da Corrente de Jato em altos níveis, a altura da troca (no caso, a altura da tropopausa) e o nível de chegada (ambas em $\mathrm{hPa}$ ) na troposfera.

O caso do dia 27 de agosto de 2013 foi selecionado para demonstrar a metodologia empregada na identificação dos eventos de TET sobre a região de interesse.

A figura 1(a) apresenta dois "cortes horizontais", um no nível de $250 \mathrm{hPa}$ a fim de mostrar a atuação da Corrente de Jato neste nível (pintados em tons de cinza) e outro em $500 \mathrm{hPa}$ (linhas contínuas $\omega>0$ e linhas tracejadas $\omega<0$ ) para indicar se há movimento ascendente ou descendente.

A parte (b) da figura 1 mostra que no dia 27 de agosto de 2013 o valor médio da coluna total de $O_{3}$ foi de 303,1 UD (Unidades Dobson), representando um aumento de
21,5\% em relação à média dos 5 dias anteriores.

$$
\text { Jet } 250 \mathrm{mb}(\mathrm{m} / \mathrm{m} / \mathrm{s}) \text { and Omega } 500 \mathrm{mb}(\mathrm{Pa} / \mathrm{s})
$$

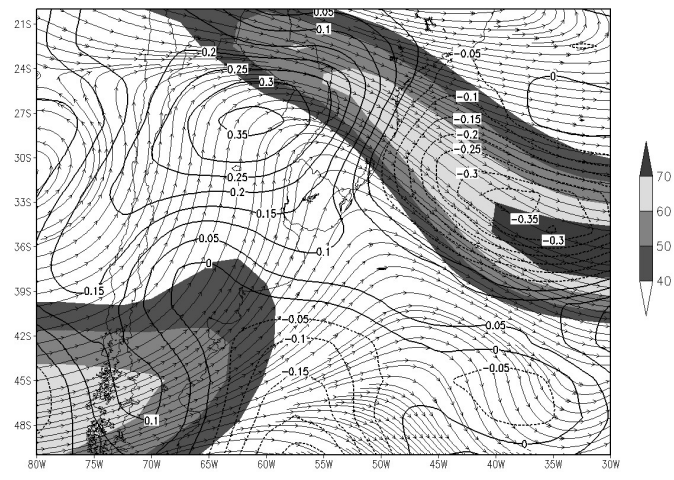

(a) Corrente de Jato em 250hPa e Omega em 500hPa;

(b)

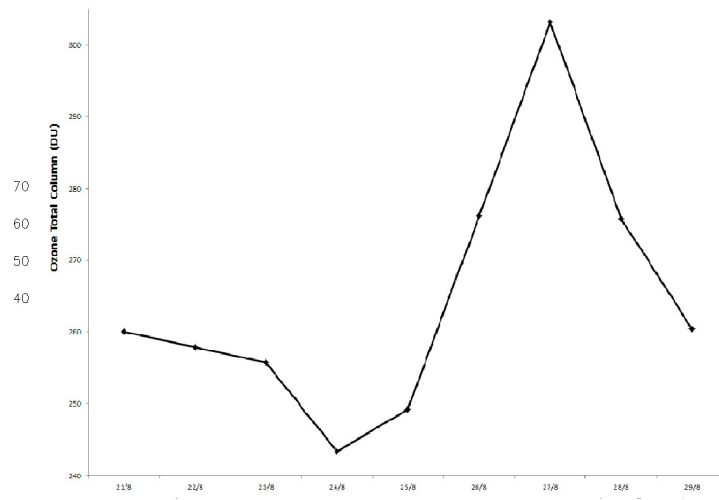

(b) Variação da Coluna Total de Ozônio (UD)

Figura 1: a)Corrente de Jato em 250hPa e Omega em $500 \mathrm{hPa}$; b) Variação da Coluna Total de Ozônio (UD) entre os dias 21 e 29 de agosto de 2013.

A figura 2(a) traz um "corte vertical" (à esquerda) de Temperatura Potencial e Vento, compreendendo a baixa estratosfera (região de maior produção/fonte de ozônio) e toda troposfera. A tropopausa aparece como a linha de 2 PVU em vermelho, denotando o limite entre as duas camadas.

A troca aconteceu aproximadamente ao longo da isentrópica de $335 \mathrm{~K}$, iniciando no nível de $250 \mathrm{hPa}$ até $700 \mathrm{hPa}$. Neste caso, também é possível observar a dobra da isolinha de $2 \mathrm{PVU}$, imediatamente ao norte da intrusão, bem como o afastamento das isentrópicas, com a atuação de uma intensa Corrente de Jato.

À direita observa-se um "corte vertical" de Fluxo de Massa (valores em azul, fluxo negativo, valores em vermelho, fluxo positivo) desde a baixa estratosfera até a baixa troposfera (chegando à CLP), com a linha de 2 PVU denotando a tropopausa. Neste caso, é notável o fluxo de massa descendente (negativo) desde $20^{\circ}$ até 


\begin{tabular}{lccc}
\multicolumn{4}{c}{ Tabela 1: Casos de TET entre 2011 e 2013. } \\
\hline TET & 2011 & 2012 & 2013 \\
\hline Casos & 4 & 5 & 4 \\
Lat. Maiores & 1 & 2 & - \\
$\begin{array}{l}\text { Lat. Iguais } \\
\text { Lat. Menores }\end{array}$ & 3 & 2 & - \\
$\begin{array}{l}\text { Corrente de Jato } \\
\text { (quantos casos) }\end{array}$ & - & 1 & Sim(2),Não(1) \\
$\begin{array}{l}\text { Altura da TET } \\
\text { (num. casos) }\end{array}$ & 200(2), 250(1), 300(1) & 200(1), 250(4) & 200(1), 250(3) \\
$\begin{array}{l}\text { Altura de Chegada } \\
\text { (num. casos) }\end{array}$ & 600(2), 900(1), 1000(1) & $600(1), 800(3), 1000(1)$ & $700(2), 1000(2)$ \\
\end{tabular}

próximo de $38^{\circ} \mathrm{S}$, cobrindo verticalmente desde o nível de $200 \mathrm{hPa}$ até próximo a superfície.

A partir destes campos, observa-se que a região de intrusão se deu na Entrada Polar do núcleo da Corrente de Jato, apresentando convergência em altos níveis e movimento descendente. Esta região está associada com forte gradiente de pressão, visto a velocidade que atinge em seu núcleo (aproximadamente $252 \mathrm{~km} / \mathrm{h}$ ). Este padrão intensifica ainda mais a descida das parcelas de ar que estão cruzando a tropopausa em $30^{\circ} \mathrm{S}$, logo ao sul do núcleo da Corrente de Jato, trazendo-as para a baixa troposfera, chegando à CLP.

Somado a isto, como visto na figura $1(\mathrm{~b})$, a coluna total de ozônio neste dia aumentou $21,5 \%$ em relação aos cinco dias anteriores, tendo então o transporte de parcelas de ar ricas em ozônio da estratosfera para a baixa troposfera, aumentando sua concentração na CLP, pelo menos nas primeiras horas após sua chegada.

Este é um exemplo dos 13 casos de TET que atuaram como fonte de ozônio para a CLP entre 2011 e 2013. É importante salientar, mais uma vez, que nestes casos preocupou-se somente com o transporte das parcelas de ar da estratosfera para dentro da baixa troposfera em dias que a coluna total de $\mathrm{O}_{3}$ aumentou em relação aos 5 dias anteriores. O que veio a ocorrer após a chegada deste composto na CLP, como mistura, diluição, etc, não foi analisado.

\section{Conclusões}

A metodologia apresentada para a deteç̧ão de TETs mostrou-se satisfatória no que diz respeito à determinação da entrada de ar estratosférico rico em ozônio para a CLP. Aconteceram 13 eventos de TET em 3 anos entre as latitudes de 20 e $50^{\circ} \mathrm{S}$ que influenciaram diretamente a entrada de ozônio estratosférico para a baixa troposfera na Região Sul do Brasil.

Destes eventos, a maioria $(69,2 \%)$ aconteceu em la-
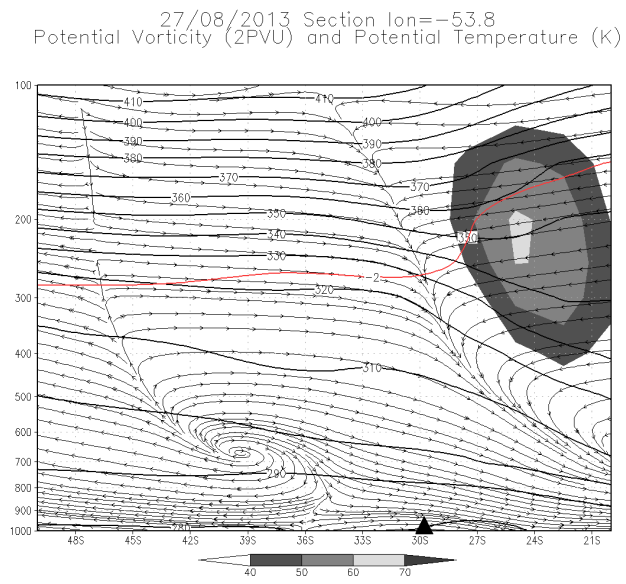

(a) Temperatura Potencial, Vento e Vorticidade Potencial Mass Flux $27 \mathrm{Kg} / \mathrm{Km}$ dia) crossing 2 PVu

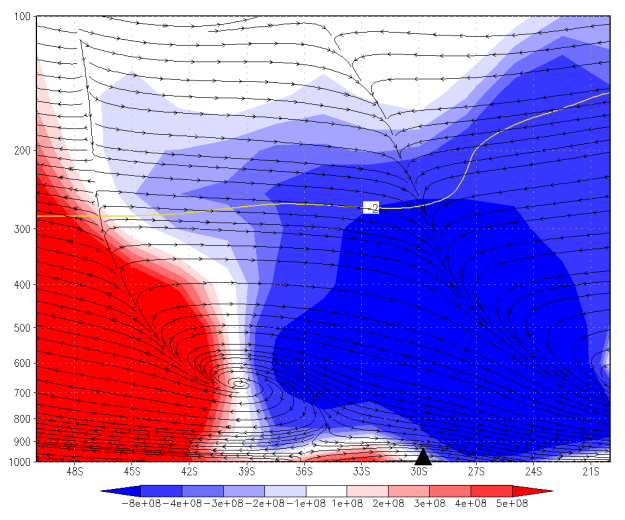

(b) Corte Vertical Fluxo de Massa

Figura 2: a)Seção transversal em $53,4^{\circ} \mathrm{O}$ de Temperatura Potencial (K), Vento $(\mathrm{m} / \mathrm{s})$ e Vorticidade Potencial $(2$ PVU em vermelho) b) Corte Vertical do Fluxo de Massa $\left(\mathrm{Kg} / \mathrm{Km}^{2}\right.$ dia cruzando a isolinha de $2 \mathrm{PVU}$ para o dia 27/08/2013.) 
titudes entre 29 e $31^{\circ} \mathrm{S}$, três casos $(23,1 \%)$ ocorreu em latitudes maiores e um caso $(7,7 \%)$ em latitude menor que esta delimitação. Este resultado era esperado, visto que a concentração de ozônio varia com a latitude, aumentando em direção aos polos; ou seja, parcelas de ar provenientes de latitudes maiores têm mais chances de conter maior conteúdo de $\mathrm{O}_{3}$.

A maioria dos casos de TET aconteceu juntamente com a atuação da Corrente de Jato $(61,5 \%)$, a qual intensifica o movimento descendente das parcelas de ar que cruzam a tropopausa em direção à troposfera, visto sua circulação vertical transversa.

Considerando que os seres vivos, em sua maioria, vivem na baixa troposfera e que são prejudicados pelo aumento da concentração de poluentes no ambiente onde estão, pode-se afirmar que o aumento de $\mathrm{O}_{3}$ na baixa troposfera (onde atua como um composto tóxico) afeta a qualidade de vida, principalmente dos seres humanos.

Neste sentido, um resultado importante diz respeito à quantidade de casos de TET $(30,7 \%)$ que chegaram em $1000 \mathrm{hPa}$, trazendo parcelas da estratosfera ricas em $\mathrm{O}_{3}$ praticamente para a superfície. Além disso, o valor médio do aumento da coluna total de $\mathrm{O}_{3}$ dos 13 casos foi $4,26 \%$.

\section{Agradecimentos}

À CAPES/FAPERGS pelo auxílio financeiro.

À UFSM e ao INPE CRS, pela formação e espaço físico.

À NASA, NOAA e NCEP/DOE pelos dados fornecidos

\section{Referências}

Appenzeler, C., Holton, J. R., Rosenlof, K. H. (1996). Seasonal variation of mass transport across the tropopause. Journal of Geophysical Research, 101, 15,07115,078 .

Bithell, M., Vaughan, G., Gray, J. J. (2000). Persistence of stratospheric ozone layers in the troposphere. Atmospheric Environment, 34(16), 2563-2570.

Bracci, A., Cristofanelli, P. C., Sprenger, M. S., Bonafè, U., Calzolari, F., Duchi, R., Laj, P., Marinoni, A., Roccato, F., Vuillermoz, E., Bonasoni, P. B. (2012). Transport of stratospheric air masses to the nepal climate observatory-pyramid (himalaya; $5079 \mathrm{~m} \mathrm{msl):} \mathrm{A}$ synoptic-scale investigation. Journal of Applied Meteorology and Climatology, 51(8), 1489-1507.

Brewer, A. W. (1949). Evidence for a world circulation provided by the measurements of helium and water vapour distribution in the stratosphere. Quarterly Journal of the Meteorology Royal Society, 75, 351-363.

Bukin, O. A., An, N. S., Pavlov, A. N., Stolyarchuk, A. Y., Shmirko, K. A. (2011). Effect that jet streams have on the vertical ozone distribution and characteristics of tropopause inversion layer. Izvestiya Atmospheric and Oceanic Physics, 47(5), 610-618.

Cristofanelli, P., Bonasoni, P., Tositti, L., Bonafè, U., Calzolari, F., Evangelisti, F., Sandrini, S., Stohl, A. (2006). A 6-year analysis of stratospheric intrusions and their influence on ozone at $\mathrm{mt}$. cimone $(2165 \mathrm{~m}$ above sea level). Journal of Geophysical Research, 111.

Danielsen, E. F. (1968). Stratospheric-tropospheric exchange based upon radioactivity, ozone and potential vorticity. Journal of the Atmospheric Science, 25, 502-518.

Davies, T. D., Schuepbach, E. (1994). Episodes of high ozone concentrations at the earth's surface resulting from transport down from the upper troposphere/lower stratosphere: a review and case studies. Atmospheric Environment, 28, 53-68.

De Bellevue, J. L., Réchou, A., Baray, J. L., Ancellet, G., Diab, R. D. (2006). Signatures of stratosphere to troposphere transport near deep convective events in the southern subtropics. Journal of Geophysical Research: Atmospheres, 111.

Dobson, G. M. (1968). Forty years' research on atmospheric ozone at oxford: A history. Applied Optics, 7, 387405.

Ertel, H. (1942). Ein neuer hydrodynamischer wirbelsatz. Meteorol Z, 59, 271-281.

Gerasopoulos, E., Prodromos, Z., Papastefanou, C., Zerefos, C. S., Ioannidou, A., Wernli, H. (2006). A complex case study of down to the surface intrusions of persistent stratospheric air over the eastern mediterranean. Atmospheric Environment, 40, 4113-4125.

Gettelman, A., Hoor, P., Pan, L. L., Randel, W. J., Hegglin, M. I., Birner, T. (2011). The extratropical upper troposphere and lower stratosphere. Reviews of Geophysics, 49(RG3003).

Haagenson, P. L., Shapiro, M. A., Middleton, P., Laird, A. R. (1981). A case study relating high ground level ozone to enhanced photochemistry and isentropic transport from the stratosphere. Journal of Geophysical Research, 86, 5231-5237.

Hocking, W. K., Carey-Smith, T., Tarasick, D. W., Argall, P. S., Strong, K., Rochon, Y., Zawadzki, I., Taylor, P. A. (2007). Detection of stratospheric ozone intrusions by wind profiler radars. Nature, 450, 281-284. 
Holton, J. R., Haynes, P. H., Douglass, A. R., Rood, R. B., Pfister, L. (1995). Stratosphere-troposphere exchange. Reviews of Geophysics, 3(3), 403-439.

Kuang, S., Newchurch, M. J., Burris, J., Wang, L., Knupp, K., Huang, G. (2012). Stratosphere-to-troposphere transport revealed by ground-based lidar and ozonesonde at a midlatitude site. Journal of Geophysical Research, 117, 18,305.

Manney, G. L., Hegglin, M. I., Daffer, W. H., Santee, M. L., Ray, E. A., Pawson, S., Schwartz, M. J., Boone, C. D., Froidevaux, L., Livesey, N. J., Read, W. G., Walker, K. A. (2011a). Jet characterization in the upper troposphere/lower stratosphere (utls): applications to climatology and transport studies. Atmospheric Chemistry and Physics, 11, 6115-6137.

NASA (2015). AURA Validation Data Center - Ozone Monitoring Instrument. National Aeronautics and Space Administration, Greenbelt, Maryland, USA, URL http://avdc.gsfc.nasa.gov/index.php?site \let

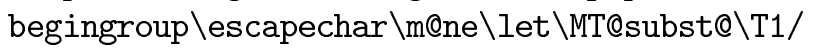
$\mathrm{pplx} / \mathrm{m} / \mathrm{it} / 10 \backslash \operatorname{def}\{\backslash @ @ \mathrm{par}\}$.

Roelofs, G. J., Lelieveld, J. (1997). Model study of the influence of cross-tropopause $o_{3}$ transports on tropospheric $o_{3}$ levels. Tellus $B, 49,38-55$.

Skerlak, B., Sprenger, M., Wernli, H. (2014). A global climatology of stratosphere-troposphere exchange using the era-interim data set from 1979 to 2011. Atmospheric Chemistry and Physics, 14, 913-937.

Skerlak, B., Sprenger, M., Pfahl, S., Tyrlis, E., Wernli, H. (2015). Tropopause folds in era-interim: Global climatology and relation to extreme weather events. Journal of Geophysical Research, 120, 4860-4877.

Tang, Q., Prather, M. J. (2010). Correlating tropospheric column ozone with tropopause folds: the aura-omi satellite data. Atmospheric Chemistry and Physics, 10, $14,875-14,896$.

Trickl, T., Bärtsch-Ritter, N., Eisele, H., Furger, M., Mücke, R., Sprenger, M., Stohl, A. (2011). igh-ozone layers in the middle and upper troposphere above central europe: potential import from the stratosphere along the subtropical jet stream. Atmospheric Chemistry and Physics, 11, 9343-9366. 